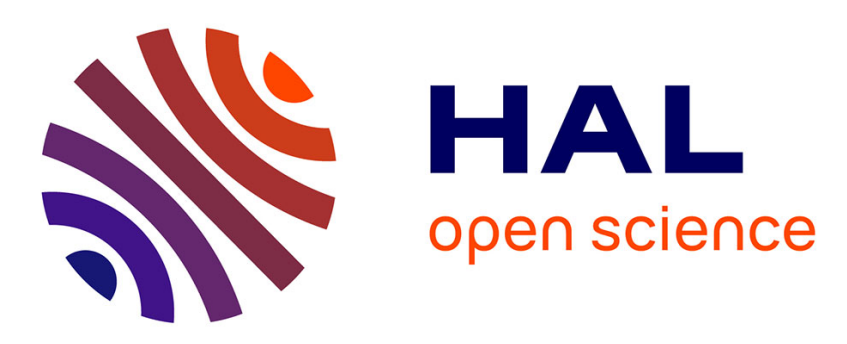

\title{
Atmospheric Chemistry Experiment (ACE): Mission overview.
}

\author{
P. F. Bernath, C.T. Mcelroy, M. C. Abrams, C.D. Boone, M. Butler, C. \\ Camy-Peyret, M. Carleer, Cathy Clerbaux, Pierre-François Coheur, R. Colin, \\ et al.
}

\section{To cite this version:}

P. F. Bernath, C.T. Mcelroy, M. C. Abrams, C.D. Boone, M. Butler, et al.. Atmospheric Chemistry Experiment (ACE): Mission overview.. Geophysical Research Letters, 2005, 32 (15), pp.L15S01. 10.1029/2005GL022386 . hal-00112746

\section{HAL Id: hal-00112746 https://hal.science/hal-00112746}

Submitted on 6 Aug 2020

HAL is a multi-disciplinary open access archive for the deposit and dissemination of scientific research documents, whether they are published or not. The documents may come from teaching and research institutions in France or abroad, or from public or private research centers.
L'archive ouverte pluridisciplinaire HAL, est destinée au dépôt et à la diffusion de documents scientifiques de niveau recherche, publiés ou non, émanant des établissements d'enseignement et de recherche français ou étrangers, des laboratoires publics ou privés. 


\title{
Atmospheric Chemistry Experiment (ACE): Mission overview
}

\author{
P. F. Bernath, ${ }^{1}$ C. T. McElroy ${ }^{2}$ M. C. Abrams,${ }^{3}$ C. D. Boone,${ }^{1}$ M. Butler, ${ }^{1}$ \\ C. Camy-Peyret, ${ }^{4}$ M. Carleer, ${ }^{5}$ C. Clerbaux,${ }^{6}$ P.-F. Coheur, ${ }^{5}$ R. Colin, ${ }^{5}$ P. DeCola, ${ }^{7}$ \\ M. DeMazière, ${ }^{8}$ J. R. Drummond,${ }^{9}$ D. Dufour, ${ }^{9}$ W. F. J. Evans,${ }^{10}$ H. Fast ${ }^{2}$ D. Fussen, ${ }^{8}$ \\ K. Gilbert, ${ }^{11}$ D. E. Jennings, ${ }^{12}$ E. J. Llewellyn, ${ }^{13}$ R. P. Lowe, ${ }^{11}$ E. Mahieu, ${ }^{14}$ \\ J. C. McConnell, ${ }^{15}$ M. McHugh, ${ }^{16}$ S. D. McLeod, ${ }^{1}$ R. Michaud, ${ }^{17}$ C. Midwinter, ${ }^{2,9}$ \\ R. Nassar, ${ }^{1}$ F. Nichitiu, ${ }^{9}$ C. Nowlan, ${ }^{9}$ C. P. Rinsland,${ }^{18}$ Y. J. Rochon, ${ }^{2}$ N. Rowlands, ${ }^{19}$ \\ K. Semeniuk,${ }^{15}$ P. Simon, ${ }^{8}$ R. Skelton, ${ }^{1}$ J. J. Sloan, ${ }^{1}$ M.-A. Soucy, ${ }^{20}$ K. Strong, ${ }^{9}$ \\ P. Tremblay, ${ }^{21}$ D. Turnbull, ${ }^{11}$ K. A. Walker, ${ }^{1}$ I. Walkty, ${ }^{22}$ D. A. Wardle, ${ }^{2}$ V. Wehrle, ${ }^{17,23}$ \\ R. Zander, ${ }^{14}$ and J. Zou ${ }^{9}$
}

Received 8 January 2005; revised 15 February 2005; accepted 22 March 2005; published 3 June 2005.

[1] SCISAT-1, also known as the Atmospheric Chemistry Experiment (ACE), is a Canadian satellite mission for remote sensing of the Earth's atmosphere. It was launched into low Earth circular orbit (altitude $650 \mathrm{~km}$, inclination $74^{\circ}$ ) on 12 Aug. 2003. The primary ACE instrument is a high spectral resolution $\left(0.02 \mathrm{~cm}^{-1}\right)$ Fourier Transform Spectrometer (FTS) operating from 2.2 to $13.3 \mu \mathrm{m}\left(750-4400 \mathrm{~cm}^{-1}\right)$. The satellite also features a dual spectrophotometer known as MAESTRO with wavelength coverage of $285-1030 \mathrm{~nm}$ and spectral resolution of $1-2 \mathrm{~nm}$. A pair of filtered CMOS detector arrays records images of the Sun at 0.525 and $1.02 \mu \mathrm{m}$. Working primarily in solar occultation, the satellite provides altitude profile information (typically $10-100 \mathrm{~km}$ ) for temperature, pressure, and the volume mixing ratios for several dozen molecules of atmospheric interest, as well as atmospheric extinction profiles over the latitudes $85^{\circ} \mathrm{N}$ to $85^{\circ} \mathrm{S}$. This paper presents a mission overview and some of the first scientific results. Citation: Bernath, P. F., et al. (2005), Atmospheric Chemistry Experiment (ACE): Mission overview, Geophys. Res. Lett., 32, L15S01, doi:10.1029/2005GL022386.

\section{Introduction}

[2] Scientific goals for ACE include: (1) understanding the chemical and dynamical processes that control the distribution of ozone in the stratosphere and upper troposphere, particularly in the Arctic; (2) exploring the rela-

\footnotetext{
${ }^{1}$ Department of Chemistry, University of Waterloo, Waterloo, Ontario, Canada.

${ }^{2}$ Meteorological Service of Canada, Environment Canada, Toronto, Ontario, Canada.

${ }^{3}$ FastMetrix Inc., Alexandria, Virginia, USA.

${ }^{4}$ Laboratoire de Physiques Moléculaire et Applications, Université Paris VI, Paris, France.

${ }^{5}$ Chimie Quantique et Photophysique, Université Libre de Bruxelles, Brussels, Belgium.

${ }^{6}$ Service d'Aeronomie, Université Paris VI, Paris, France.

${ }^{7}$ NASA Headquarters, Washington, D. C., USA.

${ }^{8}$ Belgian Institute for Space Aeronomy, Brussels, Belgium.

${ }^{9}$ Department of Physics, University of Toronto, Toronto, Ontario, Canada.

${ }^{10}$ Department of Physics, Trent University, Peterborough, Ontario,
Canada. Canada.

Copyright 2005 by the American Geophysical Union. 0094-8276/05/2005GL022386
}

tionship between atmospheric chemistry and climate change; (3) studying the effects of biomass burning on the free troposphere; and (4) measuring aerosols and clouds to reduce the uncertainties in their effects on the global energy balance. We are particularly interested in ozone chemistry because anthropogenic changes in atmospheric ozone have led to an increased amount of ultraviolet radiation reaching the ground and may also affect climate [World Meteorological Organization, 2002].

[3] A high resolution $\left(0.02 \mathrm{~cm}^{-1}\right)$ infrared Fourier transform spectrometer (ACE-FTS) operating from 750$4400 \mathrm{~cm}^{-1}$ measures the vertical distribution of trace gases and temperature. During sunrise and sunset, the FTS measures sequences of atmospheric absorption spectra in the limb viewing geometry with different slant paths and tangent heights; when these spectra are analysed, the results are inverted into vertical profiles of atmospheric constituents. The vertical resolution is about $4 \mathrm{~km}$ from the cloud tops up to about $150 \mathrm{~km}$. Aerosols and clouds are being monitored using the extinction of solar radiation as measured by two filtered imagers. A second instrument called MAESTRO (Measurement of Aerosol Extinction in the Stratosphere and Troposphere Retrieved by Occultation) was added to the original ACE mission with C. T. McElroy of the Meteorological Service of Canada (MSC) as the principal investigator. MAESTRO is a dual optical spectrophotometer that covers the $285-1030 \mathrm{~nm}$ spectral region. It has a vertical resolution of $1-2 \mathrm{~km}$ and

\footnotetext{
${ }^{11}$ Department of Physics and Astronomy, University of Western Ontario, London, Ontario, Canada.

${ }^{12}$ Planetary Systems Branch, NASA Goddard Space Flight Center, Greenbelt, Maryland, USA.

${ }^{13}$ Department of Physics and Engineering Physics, University of Saskatchewan, Saskatoon, Saskatchewan, Canada.

${ }^{14}$ Institute of Astrophysics and Geophysics, Liège, Belgium.

${ }^{15}$ Department of Earth and Space Science and Engineering, York University, Toronto, Ontario, Canada.

${ }^{16}$ GATS Inc., Newport News, Virginia, USA.

${ }^{17}$ Canadian Space Agency, Saint-Hubert, Quebec, Canada.

${ }^{18}$ NASA Langley Research Center, Hampton, Virginia, USA.

${ }^{19}$ EMS Technologies, Ottawa, Ontario, Canada.

${ }^{20}$ ABB-Bomem Inc., Quebec, Quebec, Canada.

${ }^{21}$ Departement de Genie Electrique et de Genie Informatique, Université Laval, Quebec, Quebec, Canada.

${ }^{22}$ Bristol Aerospace Ltd., Winnipeg, Manitoba, Canada.

${ }^{23}$ Now at Bristol Aerospace Ltd., Ottawa, Ontario, Canada.
} 


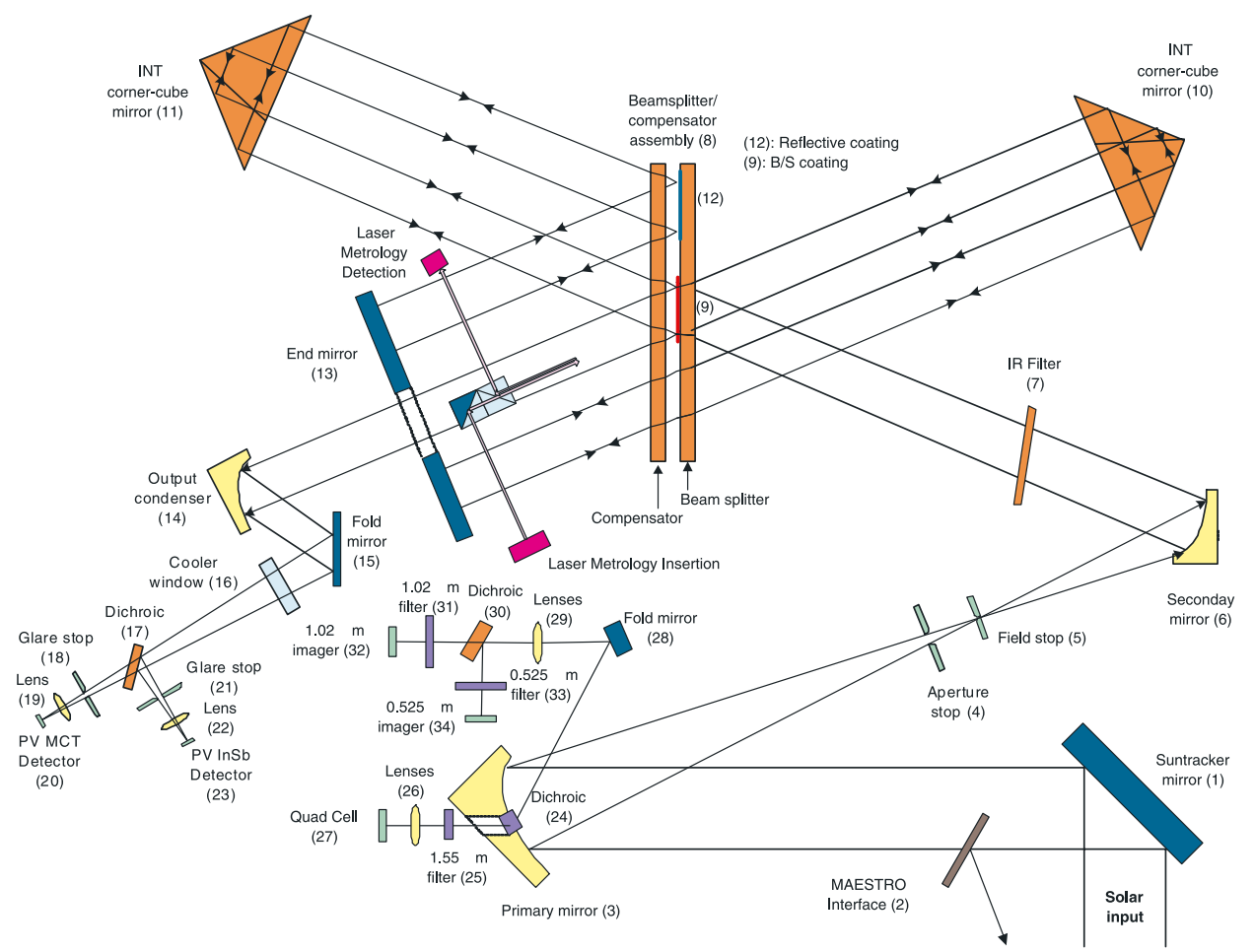

Figure 1. The optical layout of the ACE-FTS and imagers (from ABB-Bomem).

measures primarily ozone, nitrogen dioxide, and aerosol/ cloud extinction. A high inclination $\left(74^{\circ}\right)$, circular lowearth orbit $(650 \mathrm{~km})$ gives ACE coverage of tropical, midlatitude, and polar regions. ACE is the first mission in the Canadian Space Agency's (CSAs) small science satellite program.

[4] The ACE mission concept is based on the successful (but now retired) ATMOS (Atmospheric Trace MOlecule Spectroscopy) instrument that flew four times $(1985,1992$, 1993, and 1994) on the Space Shuttle [Gunson et al., 1996]. The ACE-FTS instrument has been miniaturized by nearly a factor of 10 in mass, power, and volume as compared to ATMOS. The ACE mission has been augmented by the two imagers and the spectrophotometer as compared to ATMOS. The imagers give high signal-tonoise ratio (SNR) measurements of atmospheric extinction and lead to SAGE-like aerosol and cloud data products [Kent et al., 1993]. In addition, the imagers provide an important diagnostic for the variation of the flux over the distorted solar disk in this region.

[5] The ACE-FTS, including imagers, was built by ABB-Bomem in Quebec City and the satellite bus was made by Bristol Aerospace in Winnipeg. MAESTRO was designed and built in a partnership among the MSC, the University of Toronto, and EMS Technologies in Ottawa. The satellite was launched by NASA from Vandenburg Air Force Base using a Pegasus XL rocket for a planned 2-year mission.

\section{ACE Instruments}

[6] The main instrument on SCISAT-1 is a Michelson interferometer (ACE-FTS) of a custom design. The interferometer uses two cube corners rotating on a central flex pivot to produce the optical path difference (Figure 1). An "end" mirror inside the interferometer is used to double pass the radiation and increase the optical path difference. The ACE-FTS design is fully compensated for tilt and shear of both moving and stationary optics inside the interferometer. A pointing mirror, controlled by a suntracker servo-loop, locks on the Sun center and tracks it while the instrument is taking measurements. The FTS has a circular field of view (FOV) of $1.25 \mathrm{mrad}$, a mass of about $41 \mathrm{~kg}$, and an average power consumption of $37 \mathrm{~W}$. Double-sided interferograms are Fourier transformed on the ground to obtain the desired atmospheric spectra. The FTS uses two photovoltaic detectors (InSb and $\mathrm{HgCdTe}$ ), aligned with a dichroic element to have the same field of view. The detectors are cooled to $80-100 \mathrm{~K}$ by a passive radiator pointing toward deep space.

[7] The visible/near infrared imager has two filtered channels at 0.525 and $1.02 \mu \mathrm{m}$, chosen to match two of the wavelengths monitored by the SAGE II satellite instrument [Kent et al., 1993]. The imagers also provide an important diagnostic for pointing and for detecting the presence of clouds in the FOV. The detectors in the imagers are (effectively) $128 \times 128$ active pixel sensors made by Fill Factory of Mechelen, Belgium. The total FOV of the imagers is $30 \mathrm{mrad}$, to be compared to the 9 mrad angular diameter of the Sun. The SNR of each solar image is greater than 1000 , but the main image suffers from overlap by weak secondary images from optical filters that were not tipped far enough off the optical axis.

[8] MAESTRO is a small (about $8 \mathrm{~kg}$ ) spectrophotometer that was designed to cover the $285-1030 \mathrm{~nm}$ region in two overlapping segments. The use of two spectrographs $(280-550 \mathrm{~nm}, 500-1030 \mathrm{~nm})$ reduces the stray light and 


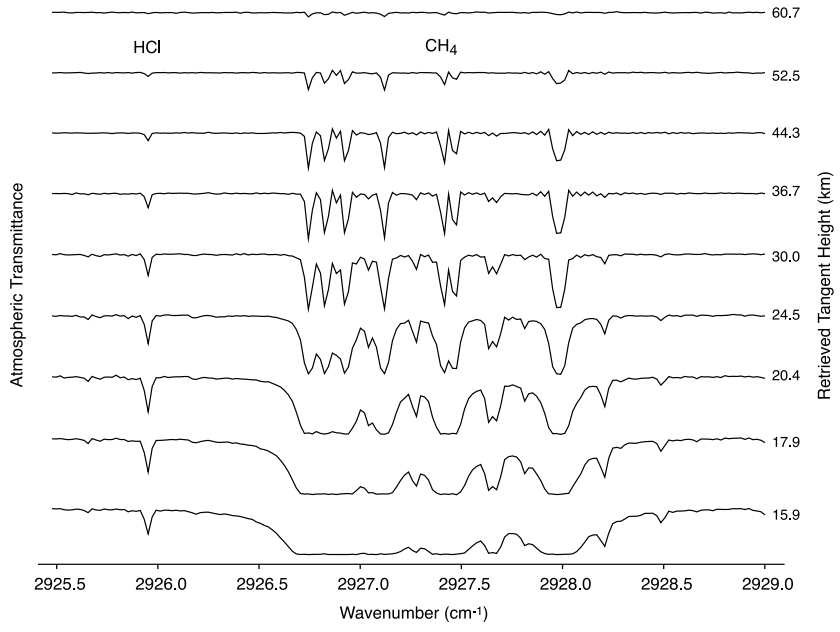

Figure 2. A small section of spectra from ACE-FTS. The $\mathrm{R}(0)$ line of the $\mathrm{HCl}$ fundamental band on the left and the cluster of $\mathrm{CH}_{4}$ lines due to $\mathrm{R}(9)$ of the $v_{3}$ band on the right. The retrieved tangent heights (in $\mathrm{km}$ ) are on the right side.

permits simultaneous measurements of the two bands with spectral resolution of $1-2 \mathrm{~nm}$, depending on wavelength. The detectors are 1024 linear EG\&G Reticon photodiode arrays. The design is based on a simple concave grating with no moving parts. The entrance slit is held horizontal with respect to the horizon during sunrise. The ACE-FTS, imagers, and MAESTRO all share a single suntracker and have approximately the same direction of view. The MAESTRO SNR is in excess of 1000. While the ACE mission will work primarily by solar occultation, MAESTRO is also able to make near-nadir solar backscatter measurements like the GOME instrument on the European ERS-2 satellite [Burrows et al., 1999].

\section{Timeline}

[9] The ACE proposal was submitted to CSA in Jan. 1998 and the ACE mission was selected for flight in November of the same year. Construction and testing of the instruments and spacecraft were largely completed by late 2002. The final testing of the instruments was carried

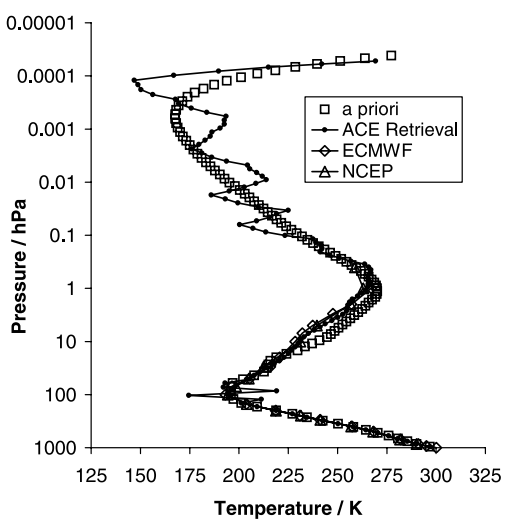

Figure 3. Comparison of the ACE-FTS a priori $T$ versus $p$ with the retrieved result and weather forecast models.

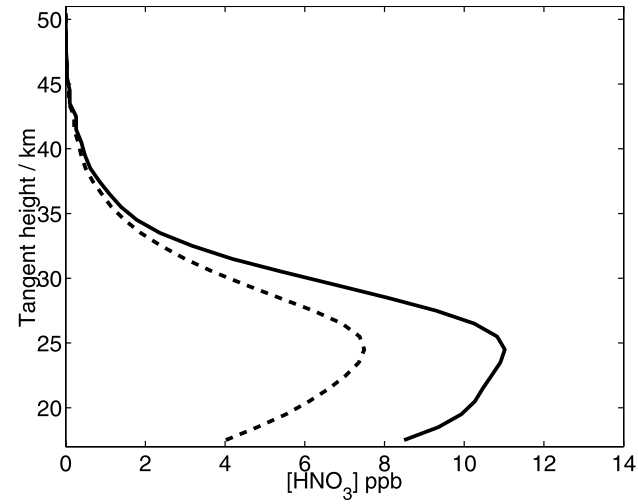

Figure 4. Averaged $\mathrm{HNO}_{3}$ VMR profile based on 104 occultations (March $1-11,77-80^{\circ} \mathrm{N}$ ) inside the Arctic vortex (solid line) and 91 mid and high latitude (March 21 April 2, $30^{\circ} \mathrm{N}-66^{\circ} \mathrm{N}$ ) occultations outside the vortex (dashed line).

out at the University of Toronto during Feb.-Mar. 2003. The launch and early orbit phase proceeded without difficulty and the first atmospheric spectra were recorded in early Dec. 2003. The spectra were of excellent quality with a SNR in excess of 300 over most of the spectral band. Only at the edges of the band (below about $900 \mathrm{~cm}^{-1}$ and above $3700 \mathrm{~cm}^{-1}$ ) does the SNR drop below 100. Science commissioning was carried out mainly in Jan. 2004, with routine operations starting in Feb. 2004.

[10] The ACE-FTS measurement sequence during a sunset occultation starts with the suntracker pointing to deep space to record a set of instrument self-emission spectra. The deep space spectra are followed by a set of high Sun reference spectra obtained by pointing to the center of the Sun, and then a sequence of occultation spectra starting at $150 \mathrm{~km}$ tangent altitude are obtained at a rate of one scan every $2 \mathrm{~s}$ as the Sun sets.

\section{Ground Segment and Data Analysis}

[11] The raw ACE data are sent to ground using two Canadian ground stations, and if all occultations were measured then the average data volume would be about 2 Gbyte/day. The current downlink capacity is about 1 Gbyte/day, but we plan to add extra downlink capacity in 2005. The raw ACE data are transferred using the internet from the Mission Operations Centre operated by CSA in St. Hubert to the Science Operations Centre at the University of Waterloo. At Waterloo the data are archived and transformed into data products for distribution to the science team members. In the case of the ACE-FTS, the raw interferograms (level 0) are transformed into corrected atmospheric spectra (level 1b) by software supplied by the instrument contractor, ABB-Bomem. The spectra are corrected for instrument self-emission and then transmission spectra are computed by dividing each atmospheric spectrum by the average high Sun spectrum. The transmission spectra show no detectable residual non-linearity or channeling effects (Figure 2), but some traces of residual solar lines remain due to small changes in the satellite-sun relative velocity during the course of an occultation. These spectra are interpolated onto a standard wavenumber grid 


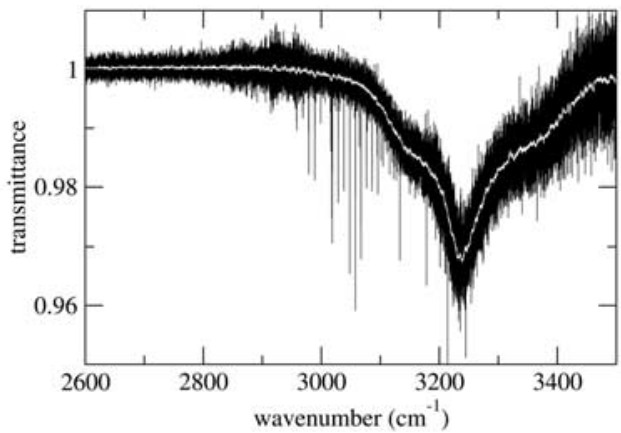

Figure 5. PMC absorption in ACE-FTS spectra. The O-H stretch feature is evident in this average of 9 spectra (black) taken during 6-14 July 2004 , near $66^{\circ} \mathrm{N}$. All spectra have tangent point altitudes near $83 \mathrm{~km}$. The result of a 128-point median filter is shown as the white line. This feature is consistent with absorption by ice particles with $\sim 50 \mathrm{~nm}$ effective radius.

and our retrievals (level 1 to 2) use selected microwindows in these raw spectra without apodization.

[12] Level 2 data are height profiles of the volume mixing ratios (VMRs) of atmospheric constituents, and are generated by analysis software developed at Waterloo. During level 1 to 2 data processing, a series of spectra measured during an occultation are used to infer altitude profiles for the atmospheric quantities of interest.

[13] The first step in the level 1 to 2 data processing is the retrieval of temperature and pressure, beginning with an a priori profile (C. D. Boone et al., Retrievals for the Atmospheric Chemistry Experiment Fourier transform spectrometer, submitted to Applied Optics, 2005, hereinafter referred to as Boone et al., submitted manuscript, 2005). Retrievals of temperature from the FTS data assume a fixed $\mathrm{CO}_{2} \mathrm{VMR}$ in the altitude range of about 10 to $70 \mathrm{~km}$ (Boone et al., submitted manuscript, 2005). In essence, the relative $\mathrm{CO}_{2}$ line intensities are used to determine the temperature and the absolute line intensities give the pressure. For the ACE-FTS, we use a global fit approach in the level 1 to 2 processing [Carlotti, 1988].

[14] Figure 3 shows the result for a temperature retrieval from an occultation measured in February, 2004. Our retrievals compare very favorably with the predictions of weather forecast models from the European Centre for Medium-Range Weather Forecasts (ECMWF) and the American National Centers for Environmental Prediction (NCEP), and have a typical accuracy of 1-2 K below $50 \mathrm{~km}$. Note that our mesospheric retrievals are not yet validated, and that Figures 2-3 are based on the data of a single tropical occultation (sunset 2551) recorded on 2 February 2004 at $9^{\circ} \mathrm{S}$ latitude $132^{\circ} \mathrm{W}$ longitude. Many of the temperature profiles (e.g., Figure 3) show evidence of atmospheric wave activity in the mesosphere.

[15] The second step in the level 1 to 2 data processing is the retrieval of altitude profiles of VMRs of gaseous atmospheric constituents. For the FTS data, selected microwindows are used to retrieve the altitude profiles [Carlotti, 1988]. We have adopted the HITRAN 2004 [Rothman et al., 2005] spectroscopic database for the line parameters and cross sections for the initial processing of ACE-FTS data. The MAESTRO algorithms draw on the previous work by C. T. McElroy and co-workers with a variety of $\mathrm{UV} /$ visible spectrographs that have been deployed, for example, on the NASA ER-2 aircraft [McElroy, 1995].

\section{Results}

[16] Version 1.0 of the ACE-FTS level 2 data has been generated at the University of Waterloo and consists of profiles of 18 molecules: $\mathrm{H}_{2} \mathrm{O}, \mathrm{O}_{3}, \mathrm{~N}_{2} \mathrm{O}, \mathrm{CO}, \mathrm{CH}_{4}, \mathrm{NO}$, $\mathrm{NO}_{2}, \mathrm{HNO}_{3}, \mathrm{HF}, \mathrm{HCl}, \mathrm{N}_{2} \mathrm{O}_{5}, \mathrm{ClONO}_{2}, \mathrm{CCl}_{2} \mathrm{~F}_{2}, \mathrm{CCl}_{3} \mathrm{~F}$, $\mathrm{COF}_{2}, \mathrm{CHF}_{2} \mathrm{Cl}, \mathrm{HDO}, \mathrm{SF}_{6}$, plus $p$ and $T$, interpolated on a $1 \mathrm{~km}$ grid. This data set consists of the results for sunsets from Feb.-Oct. 2004, and is intended primarily for validation exercises [Walker et al., 2005], but is also used for preliminary scientific investigations. These level 2 data are available at http://www.ace.uwaterloo.ca/data/. Preliminary scientific studies with the version 1.0 results include investigating the current levels of stratospheric water vapor [Nassar et al., 2005] and trends of various molecules [Rinsland et al., 2005a] (through comparison with the previous ATMOS measurements). Spring 2004 in the northern hemisphere saw a major enhancement of $\mathrm{NO}$ and $\mathrm{NO}_{2}$ levels in the stratosphere [Rinsland et al., 2005b]. ACE was fortunate enough to capture this enhancement, and we have investigated this effect in some detail. In addition, we have also found increased levels of $\mathrm{HNO}_{3}$ in the polar vortex in Mar. 2004 (Figure 4). These enhanced levels of $\mathrm{HNO}_{3}$ may simply be due to descent in the polar night and we are working on the NOy budget. We have also detected the first infrared spectra of polar mesospheric clouds (PMCs), which prove to be small ice particles as expected (Figure 5).

[17] Acknowledgments. We thank the ACE team of scientists, engineers and managers whose efforts have made the mission a success. Funding for ACE is provided by the CSA, the Natural Sciences and Engineering Research Council of Canada, the MSC, and the Canadian Foundation for Climate and Atmospheric Sciences. Support at Waterloo was also provided by the NSERC-Bomem-CSA-MSC Industrial Research Chair in Fourier Transform Spectroscopy. We also are grateful for the launch and other support provided by NASA. Support was also provided by the Belgian government for the imager detector arrays and for science.

\section{References}

Burrows, J. P., et al. (1999), The Global Ozone Monitoring Experiment (GOME): Mission concept and first scientific results, J. Atmos. Sci., 56, $151-175$.

Carlotti, M. (1988), Global-fit approach to the analysis of limb-scanning atmospheric measurements, Appl. Opt., 27, 3250-3254.

Gunson, M. R., et al. (1996), The Atmospheric Trace Molecule Spectroscopy (ATMOS) experiment: Deployment on the ATLAS space shuttle missions, Geophys. Res. Lett., 23, 2333-2336.

Kent, G. S., et al. (1993), A model for the separation of cloud and aerosol in SAGE II occultation data, J. Geophys. Res., 98, 20,725-20,735.

McElroy, C. T. (1995), A spectroradiometer for the measurement of direct and scattered solar irradiance from on-board the NASA ER-2 highaltitude research aircraft, Geophys. Res. Lett., 22, 1361-1364.

Nassar, R., P. F. Bernath, C. D. Boone, G. L. Manney, S. D. McLeod, C. P. Rinsland, R. Skelton, and K. A. Walker (2005), Stratospheric abundances of water and methane based on ACE-FTS measurements, Geophys. Res. Lett., 32, L15S04, doi:10.1029/2005GL022383.

Rinsland, C. P., C. Boone, R. Nassar, K. Walker, P. Bernath, E. Mahieu, R. Zander, J. C. McConnell, and L. Chiou (2005a), Trends of HF, HCl, $\mathrm{CCl}_{2} \mathrm{~F}_{2}, \mathrm{CCl}_{3} \mathrm{~F}, \mathrm{CHClF}_{2}$ (HCFC-22), and $\mathrm{SF}_{6}$ in the lower stratosphere from Atmospheric Chemistry Experiment (ACE) and Atmospheric Trace Molecule Spectroscopy (ATMOS) measurements near $30^{\circ} \mathrm{N}$ latitude, Geophys. Res. Lett., doi:10.1029/2005GL022415, in press.

Rinsland, C. P., C. Boone, R. Nassar, K. Walker, P. Bernath, J. C. McConnell, and L. Chiou (2005b), Atmospheric Chemistry Experiment (ACE) Arctic stratospheric measurements of $\mathrm{NO}_{\mathrm{x}}$ and long-lived tracers 
during February and March 2004: Impact of intense solar flares, Geophys. Res. Lett., doi:10.1029/2005GL022425, in press.

Rothman, L. S., et al. (2005), The HITRAN 2004 molecular spectroscopic database, J. Quant. Spectrosc. Radiat. Transfer, in press.

Walker, K. A., C. E. Randall, C. R. Trepte, C. D. Boone, and P. F. Bernath (2005), Initial validation comparisons for the Atmospheric Chemistry Experiment (ACE-FTS), Geophys. Res. Lett., doi:10.1029/ 2005GL022388, in press.

World Meteorological Organization (2002), Scientific assessment of ozone depletion: 2002, Global Ozone Res. Monit. Proj. Rep. 47, Geneva, Switzerland.

M. C. Abrams, FastMetrix Inc., Alexandria, VA, USA

P. F. Bernath, C. D. Boone, M. Butler, S. D. McLeod, R. Nassar, R. Skelton, J. J. Sloan, and K. A. Walker, Department of Chemistry, University of Waterloo, 200 University Ave. W, Waterloo, ON, Canada N2L 3G1. (bernath@uwaterloo.ca)

C. Camy-Peyret, Laboratoire de Physiques Moléculaire et Applications, Université Paris VI, Paris, France.

M. Carleer, P.-F. Coheur, and R. Colin, Chimie Quantique et Photophysique, Université Libre de Bruxelles, Brussels, Belgium.

C. Clerbaux, Service d'Aeronomie, Université Paris VI, Paris, France.

P. DeCola, NASA Headquarters, Washington, D. C., USA

M. DeMazière, D. Fussen, and P. Simon, Belgian Institute for Space Aeronomy, Brussels, Belgium.
J. R. Drummond, D. Dufour, F. Nichitiu, C. Nowlan, K. Strong, and J. Zou, Department of Physics, University of Toronto, Toronto, Ontario, Canada.

W. F. J. Evans, Department of Physics, Trent University, Peterborough, Ontario, Canada.

H. Fast, C. T. McElroy, C. Midwinter, Y. J. Rochon, and D. A. Wardle, Meteorological Service of Canada, Environment Canada, Toronto, Ontario, Canada.

K. Gilbert, R. P. Lowe, and D. Turnbull, Department of Physics and Astronomy, University of Western Ontario, London, Ontario, Canada.

D. E. Jennings, Planetary Systems Branch, NASA Goddard Space Flight Center, Greenbelt, MD, USA

E. J. Llewellyn, Department of Physics and Engineering Physics, University of Saskatchewan, Saskatoon, Saskatchewan, Canada.

E. Mahieu and R. Zander, Institute of Astrophysics and Geophysics, Liège, Belgium.

J. C. McConnell and K. Semeniuk, Department of Earth and Space Science and Engineering, York University, Toronto, Ontario, Canada.

M. McHugh, GATS Inc., Newport News, VA, USA.

R. Michaud, Canadian Space Agency, Saint-Hubert, Quebec, Canada.

C. P. Rinsland, NASA Langley Research Center, Hampton, VA, USA.

N. Rowlands, EMS Technologies, Ottawa, Ontario, Canada.

M.-A. Soucy, ABB-Bomem Inc., Quebec, Quebec, Canada.

P. Tremblay, Departement de Genie Electrique et de Genie Informatique, Université Laval, Quebec Quebec, Canada.

I. Walkty, Bristol Aerospace Ltd., Winnipeg, Manitoba, Canada.

V. Wehrle, Bristol Aerospace Ltd., Ottawa, Ontario, Canada 